\title{
Quantum Algebra Structure of Certain Jackson Integrals
}

\author{
Atsushi Matsuo \\ Department of Mathematics, Nagoya University, Nagoya 464-01, Japan
}

Received: June 29, 1992; in revised form February 19, 1993

\begin{abstract}
The $q$-difference system satisfied by Jackson integrals with a configuration of $A$-type root system is studied. We explicitly construct some linear combination of Jackson integrals, which satisfies the quantum Knizhnik-Zamolodchikov equation for the 2-point correlation function of $q$-vertex operators, introduced by Frenkel and Reshetikhin, for the quantum affine algebra $U_{q}\left(\widehat{\mathfrak{s l}}_{2}\right)$. The expression of integrands for the $n$-point case is conjectured, and a set of linear relations for the corresponding Jackson integrals is proved.
\end{abstract}

\section{Introduction}

1.1. In a recent work [FR], Frenkel and Reshetikhin constructed a $q$-analogue of the vertex operators of Tsuchiya and Kanie [TK] and derived a $q$-difference equation for the $n$-point correlation function, which is a $q$-analogue of the Knizhnik-Zamolodchikov equation (KZ) in the Wess-Zumino-Witten model and is called the quantum Knizhnik-Zamolodchikov equation ( $\mathrm{qKZ}$ ). This equation is written in terms of the trigonometric $\mathrm{R}$-matrices arising from finite dimensional representations of the quantum affine algebra $U_{q}(\hat{\mathrm{g}})$ (cf. [J1]) corresponding to a simple Lie algebra $\mathfrak{g}$, and plays an essential role to produce elliptic solutions of the quantum Yang-Baxter equation (YBE) of IRF type. One needs to solve the connection problem of $\mathrm{qKZ}$ to obtain an explicit form of the elliptic solutions of YBE in this context.

Therefore to indicate solutions of $\mathrm{qKZ}$ explicitly is of great importance to consider the following question: Do all the known elliptic solutions of YBE of IRF type come from the connection matrix of qKZ? Actually in the simplest case for $U_{q}\left(\widehat{s}_{2}\right)$, Heine's basic hypergeometric functions with Jackson integral representations are used to represent solutions to $\mathrm{qKZ}$ for 2-pont function in [FR] and the resulting connection matrix coincides with the $\mathrm{ABF}$-solution of YBE (cf. [ABF, JMO]).

In a previous paper [Ma2], we have constructed a Jackson integral solution to $\mathrm{qKZ}$ for the $n$-point function of the $q$-vertex operators of $U_{q}\left(\widehat{\mathfrak{s l}}_{2}\right)$ for certain kinds 
of representations provided the number of integration variables is one. The aim of this paper is to generalize it to multiple cases. We conjecture an explicit expression of a solution and prove it for the 2-point case. Our result is analogous to the case of ordinary $\mathrm{KZ}$ ( $q=1$ case), while the proofs are much more technical.

In Subsects. 1.2-1.4 below, let us briefly describe our method comparing it with that of the $q=1$ case.

1.2. Let us review the result of [DJMM] on an integral representation of solutions to $\mathrm{KZ}$ for $\widehat{\mathfrak{s l}}_{2}$. The simple Lie algebra $\mathfrak{g}=\widehat{\mathfrak{s}}_{2}$ is generated by three elements $X^{ \pm}$, $H$ with the relation

$$
\left[X^{+}, X^{-}\right]=H, \quad\left[H, X^{ \pm}\right]= \pm 2 X^{ \pm} .
$$

Let $\pi_{i}: \mathfrak{g} \rightarrow \operatorname{End}\left(V_{i}\right), i=0, \ldots, n, \infty$ be a set of irreducible representations with the highest weight vectors $v_{i}$ of the weights $\lambda_{i}$. We assume, for some positive integer $m$, that

$$
\lambda_{0}+\cdots+\lambda_{n}-\lambda_{\infty}=m \alpha,
$$

where $\alpha$ stands for the simple root of $g$. We consider the tensor product $V_{0} \otimes \cdots \otimes V_{n}$ and define the quadratic Casimir operator $\Omega_{i j} \in$ End $\left(V_{0} \otimes \cdots \otimes V_{n}\right)$ by

$$
\Omega_{i j}=\left(\pi_{i} \otimes \pi_{j}\right)\left(X^{+} \otimes X^{-}+X^{-} \otimes X^{+}+\frac{1}{2} H \otimes H\right) .
$$

Let $\vec{k}=\left(k_{0}, \ldots, k_{n}\right)$ be a multi-index such that $k_{0}+\cdots+k_{n}=m$. Put $a_{i}=k_{0}+\cdots+k_{i}$. For such a multi-index $\vec{k}$, we associate a vector $v_{\vec{k}} \in V_{0} \otimes \cdots \otimes V_{n}$ and a rational function $\tilde{\varphi}_{\vec{k}}(x, t)=\tilde{\varphi}_{\vec{k}}\left(x_{0}, \ldots, x_{n}, t_{1}, \ldots, t_{m}\right)$ as follows:

$$
\begin{aligned}
v_{\vec{k}} & =\frac{\left(X^{-}\right)^{k_{0}}}{k_{0} !} v_{0} \otimes \cdots \otimes \frac{\left(X^{-}\right)^{k_{n}}}{k_{n} !} v_{n}, \\
\tilde{\varphi}_{\vec{k}}(x, t) & =\sum_{w \in S_{m}} \prod_{i=0}^{n}\left(\prod_{a_{i-1}+1 \leqq j \leqq a_{i}} \frac{1}{t_{w(j)}-x_{i}}\right) .
\end{aligned}
$$

Here $S_{m}$ denotes the symmetric group acting on $m$ letters $\{1, \ldots, m\}$. Let $v$ be a complex parameter, which is related to the level $l$ of representations of the affine Lie algebra $\hat{\mathrm{g}}=\widehat{\mathfrak{s}}_{2}$ by $v=\frac{1}{2(l+2)}$. We set

$$
\begin{aligned}
\Phi(x, t)= & \prod_{0 \leqq i<j \leqq n}\left(x_{i}-x_{j}\right)^{2\left(\lambda_{i}, \lambda_{j}\right) v} \\
& \times \prod_{\substack{0 \leqq i \leqq n \\
1 \leqq j \leqq m}}\left(x_{i}-t_{j}\right)^{-2\left(\lambda_{i}, \alpha\right) v} \prod_{1 \leqq i<j \leqq m}\left(t_{i}-t_{j}\right)^{2(\alpha, \alpha) v},
\end{aligned}
$$

and define

$$
\mathscr{F}=\sum_{\vec{k}}\left(\int \Phi(x, t) \tilde{\varphi}_{\vec{k}}(x, t) d t_{1} \ldots d t_{m}\right) v_{\vec{k}} .
$$

Here the contour is appropriately chosen. Then we have 
Proposition 1.2. The vector $\mathscr{F}$ is a highest weight vector in $V_{0} \otimes \cdots \otimes V_{n}$ and satisfies the $K Z$ :

$$
\frac{\partial}{\partial x_{i}} \mathscr{F}=2 v \sum_{\substack{0 \leqq j \leqq n \\ j \neq i}} \frac{\Omega_{i j}}{x_{i}-x_{j}} \mathscr{F}, \quad i=0, \ldots, n .
$$

The construction above is an interpretation of a result of Aomoto [A1]. A generalization to the $\mathfrak{s l}_{n}$ case is given by the author [Ma1]. Similar integral solutions for an arbitrary simple Lie algebra $g$ are constructed by Schechtman and Varchenko [SV1, SV2]. Note that $[\mathrm{ZF}]$ and $[\mathrm{CF}]$ are pioneering works on this direction and see also [K] and [ATY].

1.3. Let us consider a trigonometric analogue of the last construction. We keep the notations in 1.2, except

$$
\tilde{\varphi}_{\vec{k}}(x, t)=\sum_{w \in S_{m}} \prod_{i=0}^{n}\left(\prod_{a_{i-1}+1 \leqq j \leqq a_{i}} \frac{x_{i}}{x_{i}-t_{w(j)}}\right)
$$

and

$$
\begin{aligned}
\Phi(x, t)= & \prod_{\substack{1 \leqq i \leqq n \\
1 \leqq j \leqq m}}\left(\frac{x_{i}}{t_{j}}\right)^{-\left(\lambda_{i}, \alpha\right) v}\left(1-\frac{t_{j}}{x_{i}}\right)^{-2\left(\lambda_{i}, \alpha\right) v} \\
& \times \prod_{1 \leqq i<j \leqq m}\left(\frac{t_{j}}{t_{i}}\right)^{(\alpha, \alpha) v}\left(1-\frac{t_{i}}{t_{j}}\right)^{2(\alpha, \alpha) v} \prod_{1 \leqq i \leqq n} x_{i}^{-\left(\lambda_{i}, \alpha\right) v} \prod_{1 \leqq j \leqq m} t_{j}^{\left(\lambda_{0}+\lambda_{\infty}+\alpha, \alpha\right) v} .
\end{aligned}
$$

Using them we define $\mathscr{F}$ by.

$$
\mathscr{F}=\sum_{\vec{k}}\left(\int \Phi(x, t) \tilde{\varphi}_{\vec{k}}(x, t) \frac{d t_{1}}{t_{1}} \cdots \frac{d t_{m}}{t_{m}}\right) v_{\vec{k}} .
$$

Now we formally put $x_{0}=\infty$ and define

$$
r_{i j}(x)=-\frac{x_{i}+x_{j}}{x_{i}-x_{j}}\left(\Omega_{i j}-\frac{1}{2}\left(\lambda_{i}, \lambda_{j}\right)\right)+\left(\pi_{i} \otimes \pi_{j}\right)\left(X^{+} \otimes X^{-}-X^{-} \otimes X^{+}\right) .
$$

They form a set of classical $r$-matrices normalized as:

$$
r_{i j}(x) v_{i} \otimes v_{j}=0 \text {. }
$$

For a weight $\lambda$, we define

$$
\pi_{i}(\lambda)\left(X^{-}\right)^{k} v_{i}=\left(\lambda, \lambda_{i}-k \alpha\right)\left(X^{-}\right)^{k} v_{i}
$$

Then we have

Proposition 1.3. The vector $\mathscr{F}$ is a highest weight vector in $V_{0} \otimes \cdots \otimes V_{n}$ and satisfies the trigonometric $K Z$ :

$$
x_{i} \frac{\partial}{\partial x_{i}} \mathscr{F}=v\left(\sum_{\substack{0 \leqq j \leqq n \\ j \neq \neq}} r_{i j}(x)+\pi_{i}\left(\lambda_{\infty}+\alpha\right)-\left(\lambda_{\infty}, \lambda_{i}\right)\right) \mathscr{F}, \quad i=1, \ldots, n .
$$

This construction is due to Cherednik [Ch], where integral solutions to the trigonometric $\mathrm{KZ}$ for an arbitrary simple Lie algebra $g$ are given with a beautiful derivation of an explicit form $\tilde{\varphi}_{\vec{k}} v_{\vec{k}}$. 
1.4. Let us explain an approach to obtain a $q$-analogue of the constructions above. Let $U_{q}\left(\widehat{\mathfrak{s}}_{2}\right)$ be the quantum affine algebra of type $A_{1}^{(1)}$ (see 2.1). Let $\pi_{i}: \mathfrak{g} \rightarrow \operatorname{End}\left(V_{i}\right)$, $i=0, \ldots, n, \infty$ be a set of irreducible representations of the subalgebra $U_{q}\left(\mathfrak{s I}_{2}\right)$ with the highest weight vector $v_{i}$ of the weight $\lambda_{i}$. Let $R_{V_{i} V_{j}}(x)$ be the trigonometric quantum R-matrix of $U_{q}\left(\widehat{\mathfrak{S}}_{2}\right)$ acting on $V_{i} \otimes V_{j}$, whose classical limit coincides with (1.3.4). We put $p^{-v}=q$. Then $\mathrm{qKZ}$, in a certain convention, is the following system of $q$-difference equations for a $V_{0} \otimes \cdots \otimes V_{n}$-valued function $\mathscr{F}$ :

$$
\begin{aligned}
T_{i} \mathscr{F}= & R_{V_{i} V_{i-1}}\left(\frac{p x_{i}}{x_{i-1}}\right) \cdots R_{V_{i} V_{0}}\left(\frac{p x_{i}}{x_{0}}\right) \\
& \times q^{\pi_{i}\left(\lambda_{\infty}+\alpha\right)-\left(\lambda_{\infty}, \lambda_{i}\right)} R_{V_{n} V_{i}}\left(\frac{x_{n}}{x_{i}}\right)^{-1} \cdots R_{V_{i+1} V_{i}}\left(\frac{x_{i+1}}{x_{i}}\right)^{-1} \mathscr{F}, \\
i= & 1, \ldots, n,
\end{aligned}
$$

where $T_{i}$ denotes the shift operator: $T_{i} F\left(x_{1}, \ldots, x_{n}\right)=F\left(x_{1}, \ldots, p x_{i}, \ldots, x_{n}\right)$. Here we have also put $x_{0}=\infty$.

On the other hand, a $q$-difference deformation of the function $\Phi(x, t)$ is known as follows, provided we shall here use $p$ instead of $q$. Let $(a ; p)_{\infty}$ denote $\prod_{n=0}^{\infty}\left(1-a p^{n}\right)$. Then a $q$-analogue of $(1-u)^{2 \beta}$ is given by $\frac{\left(p^{\gamma^{\prime}} u ; p\right)_{\infty}}{\left(p^{\gamma} u ; p\right)_{\infty}}$, where $\gamma-\gamma^{\prime}=\beta$. By the $q$-binomial theorem, we see that letting $p \rightarrow 1$ it tends to $(1-x)^{2 \beta}$ in a certain sense. Therefore starting from the trigonometric counterpart (1.3.2), it is natural to define

$$
\begin{aligned}
\Phi_{p}(x, t)= & \prod_{\substack{1 \leqq i \leqq n \\
1 \leqq j \leqq m}}\left(\frac{x_{i}}{t_{j}}\right)^{-\left(\lambda_{i}, \alpha\right) v} \frac{\left(p^{\left(\lambda_{i}, \alpha\right) v} t_{j} / x_{i} ; p\right)_{\infty}}{\left(p^{-\left(\lambda_{i}, \alpha\right) v} t_{j} / x_{i} ; p\right)_{\infty}} \\
& \times \prod_{1 \leqq i<j \leqq m}\left(\frac{t_{j}}{t_{i}}\right)^{(\alpha, \alpha) v} \frac{\left(p^{-(\alpha, \alpha) v} t_{i} / t_{j} ; p\right)_{\infty}}{\left(p^{(\alpha, \alpha) v} t_{i} / t_{j} ; p\right)_{\infty}} \prod_{1 \leqq i \leqq n} x_{i}^{-\left(\lambda_{i}, \alpha\right) v} \prod_{1 \leqq j \leqq m} t_{j}^{\left(\lambda_{0}+\lambda_{\infty}+\alpha, \alpha\right) v} .
\end{aligned}
$$

Instead of the integration, we use its $q$-analogue called the Jackson integral defined by

$$
\int_{0}^{s \infty} \phi(u) \frac{d_{p} u}{u}=(1-p) \sum_{n=-\infty}^{\infty} \phi\left(s p^{n}\right)
$$

for a value $s \in \mathbf{C}$.

In the present paper, we make a special selection of a set of $\tilde{\varphi}_{\vec{k}}$ which is a $q$ deformation of (1.3.1), and define a $V_{0} \otimes \cdots \otimes V_{n}$-valued function $\mathscr{F}$ in a similar way to (1.3.3), such that some analogy of the construction of Subsect. 1.2 or 1.3 would be established. The key idea is utilization of a natural $q$-deformation of the permutation used in the definition (1.2.5) or (1.3.1). To be more precise, we adopt the following action of the simple transposition $\sigma_{i}$ on a rational function:

$$
\left(\sigma_{i} f\right)\left(t_{1}, \ldots, t_{m}\right)=f\left(t_{1}, \ldots, t_{i+1}, t_{i}, \ldots, t_{m}\right) \frac{t_{i}-q^{2} t_{i+1}}{q^{2} t_{i}-t_{i+1}} .
$$

For an appropriate choice of rational functions $\varphi_{\vec{k}}$ and vectors $v_{\vec{k}} \in V_{0} \otimes \cdots \otimes V_{n}$ (see ((4.3.3) and (5.1.1) respectively), we define as follows:

$$
\tilde{\varphi}_{\vec{k}}(x, t)=\sum_{w \in S_{m}}\left(w \varphi_{\vec{k}}\right)(x, t)
$$


and

$$
\mathscr{F}=\sum_{\vec{k}}\left(\int \Phi_{p}(x, t) \tilde{\varphi}_{\vec{k}}(x, t) \frac{d_{p} t_{1}}{t_{1}} \cdots \frac{d_{p} t_{m}}{t_{m}}\right) v_{\vec{k}} .
$$

Then our main result is the following (Proposition 5.1.1 and Theorem 5.2.1):

Theorem 1.4. The vector $\mathscr{F}$ is a highest weight vector in $V_{0} \otimes \cdots \otimes V_{n}$. If $m=1$ and $n$ is arbitrary, or $n=2$ and $m$ is arbitrary, it satisfies qKZ.

1.5. The paper is organized as follows. In Sect. 2 we review the notion of a quantum affine algebra $U_{q}\left(\widehat{\mathfrak{s l}}_{2}\right)$ and the trigonometic R-matrix. We also review the definition of $\mathrm{qKZ}$. Section 3 is devoted to explain the action of the symmetric group and to prepare some technical lemmas. In Sect. 4 we choose rational functions $\tilde{\varphi}_{\vec{k}}$ and derive a set of linear relations among the corresponding Jackson integrals. In Sect. 5 we interpret the linear relations in the context of $U_{q}\left(\mathfrak{s l}_{2}\right)$, and conjecture an explicit form of a Jackson integral solution to $\mathrm{qKZ}$, which is proved to be true when $n=2$ and $m$ is arbitrary.

1.6. After completing this work the author learned that K. Mimachi [Mi2] independently studied the q-difference equation for the same kind of Jackson integrals (for $n=2$ in the present notation). He also learned that N. Reshetikhin [R] constructed a Jackson integral solution to the quantum Knizhnik-Zamolodchikov equation at least for $U_{q}\left(\widehat{s}_{2}\right)$ using a method in Bethe Ansatz.

\section{Quantum Knizhnik-Zamolodchikov Equation for $U_{q}\left(\widehat{\mathfrak{s}}_{2}\right)$}

2.1. The quantum affine algebra $\hat{U}_{q}=U_{q}\left(\widehat{\mathfrak{s l}}_{2}\right)$ is defined as an algebra over $\mathbf{C}$ with the generators:

$$
X_{0}^{ \pm}, X_{1}^{ \pm}, K_{0}^{ \pm 1}, K_{1}^{ \pm 1}
$$

and the relations:

$$
\begin{aligned}
& K_{0} K_{1}=K_{1} K_{0}, \quad K_{0} K_{0}^{-1}=K_{1} K_{1}^{-1}=1, \\
& K_{i} X_{i}^{ \pm} K_{i}^{-1}=q^{ \pm 2} X_{i}^{ \pm}, \quad K_{i} X_{j}^{ \pm} K_{i}^{-1}=q^{\mp 2} X_{j}^{ \pm} \quad(i \neq j), \\
& {\left[X_{i}^{+}, X_{j}^{-}\right] }=\delta_{i j} \frac{K_{i}-K_{i}^{-1}}{q-q^{-1}}, \\
&\left(X_{i}^{ \pm}\right)^{3} X_{j}^{ \pm}-\left(q^{2}+1+q^{-2}\right)\left(X_{i}^{ \pm}\right)^{2} X_{j}^{ \pm} X_{i}^{ \pm} \\
&+\left(q^{2}+1+q^{-2}\right) X_{i}^{ \pm} X_{j}^{ \pm}\left(X_{i}^{ \pm}\right)^{2}-X_{j}^{ \pm}\left(X_{i}^{ \pm}\right)^{3}=0 \quad(i \neq j) .
\end{aligned}
$$

Here $q$ denotes a general complex parameter. In this paper, the comultiplication $\Delta: \hat{U}_{q} \rightarrow \hat{U}_{q} \otimes \hat{U}_{q}$ is defined by

$$
\begin{aligned}
& \Delta\left(X_{i}^{+}\right)=X_{i}^{+} \otimes K_{i}+1 \otimes X_{i}^{+}, \\
& \Delta\left(X_{i}^{-}\right)=X_{i}^{-} \otimes 1+K_{i}^{-1} \otimes X_{i}^{-}, \quad \Delta\left(K_{i}\right)=K_{i} \otimes K_{i} .
\end{aligned}
$$

Let $\Delta^{(n)}$ denote the $n^{\text {th }}$ composition of the comultiplication. We put $\Delta^{\prime}=\sigma \circ \Delta$, where $\sigma(a \otimes b)=b \otimes a$ in $\hat{U}_{q} \otimes \hat{U}_{q}$. 
We consider the subalgebra $U_{q}=U_{q}\left(\mathfrak{s l}_{2}\right)$ generated by $X^{ \pm}=X_{1}^{ \pm}, K^{ \pm}=K_{1}^{ \pm}$. For each $x \in \mathbf{C}^{x}$, we define an algebra homomorphism, $\varphi_{x}: \hat{U}_{q} \rightarrow U_{q}$ by

$$
\begin{aligned}
& \varphi_{x}\left(X_{0}^{ \pm}\right)=x^{ \pm 1} X^{\mp}, \quad \varphi_{x}\left(X_{1}^{ \pm}\right)=X^{ \pm}, \\
& \varphi_{x}\left(K_{0}\right)=K^{-1}, \quad \varphi_{x}\left(K_{1}\right)=K .
\end{aligned}
$$

Let $\left(V_{i}, \pi_{i}\right)$ be a highest weight representations of $U_{q}$. Then $\left(V_{i}(x), \hat{\pi}_{i}\right)=$ $\left(V_{i}, \pi_{i}^{\circ} \varphi_{x}\right)$ gives a representation of $\hat{U}_{q}$ for each $x \in \mathbf{C}$.

Proposition 2.1.1. There exists an operator

$$
R_{V_{i} V_{j}}(x): V_{i}(x) \otimes V_{j}(1) \rightarrow V_{i}(x) \otimes V_{j}(1)
$$

whose matrix elements are rational functions in $x$ such that it satisfies the intertwining property

$$
\Delta^{\prime}(a) R_{V_{i} V_{j}}(x)=R_{V_{i} V_{j}}(x) \Delta(a), \quad a \in \hat{U}_{q},
$$

and the quantum Yang-Baxter equation:

$$
R_{V_{i} V_{j}}(x) R_{V_{i} V_{k}}(x y) R_{V_{j} V_{k}}(y)=R_{V_{j} V_{k}}(y) R_{V_{i} V_{k}}(x y) R_{V_{i} V_{j}}(x)
$$

on $V_{i} \otimes V_{j} \otimes V_{k}$.

This proposition follows, for example, from the existence of the universal $\mathrm{R}$-matrix in a certain sense ([D, T $]$ ). We can easily see that it can act on $V_{i}(x) \otimes V_{j}(1)$ as a formal power series in $x$, since $V_{i}$ is the highest weight $U_{q}$-module. Moreover normalizing by a scalar factor we obtain such an $R_{V_{i} V_{j}}(x)$ whose matrix elements are rational functions in $x$. We also refer to [FR].

2.2. We will derive in this subsection some recurrence relations for matrix elements of the R-matrix $R_{V_{i} V_{j}}(x)$.

Let $v_{i}$ be the highest weight vector in $V_{i}$ with the highest weight $\lambda_{i}$, and put

$$
M_{i}=\left(\lambda_{i}, \alpha\right) \text {. }
$$

We define

$$
v_{i}^{(k)}=\frac{\left(X^{-}\right)^{k}}{[k] !} v_{i},
$$

where the following notations are used:

$$
\begin{aligned}
{[a] } & =\frac{q^{a}-q^{-a}}{q-q^{-1}} \text { for a complex number } a, \\
{[k] ! } & =[k][k-1] \cdots[1] \text { for an integer } k
\end{aligned}
$$

Then we have

$$
\begin{aligned}
K v_{i}^{(k)}= & q^{M_{i}-2 k} v_{i}^{(k)}, \quad X^{+} v_{i}^{(k)}=\left[M_{i}-k+1\right] v_{i}^{(k-1)}, \\
& X^{-} v_{i}^{(k)}=[k+1] v_{i}^{(k+1)} .
\end{aligned}
$$

Consider the tensor product $V_{i}(x) \otimes V_{j}(1)$, which equals to $V_{i} \otimes V_{j}$ as $U_{q^{-}}$ module. For simplicity, put $i=1, j=2$ and denote $v_{1}(x) \otimes v_{2}(1) \in$ by $v_{i}^{(k)} \otimes v_{j}^{(l)}$. Let $R_{V_{1} V_{2}}(x)$ be an operator satisfying (2.1.6). For each set of integers $k, l, m$ such that $0 \leqq k \leqq m$ and $0 \leqq l \leqq m$, we define $R_{k, l}^{m}=R_{k, l}^{m}(x)$ by the following rule:

$$
R_{V_{i} V_{j}}(x) v^{(k)} \otimes v^{(m-k)}=\sum_{l=0}^{m} R_{k, l}^{m} v^{(l)} \otimes v^{(m-l)} .
$$

Otherwise we put $R_{k, l}^{m}=0$. 
Lemma 2.2.1. $R_{k, l}^{m}$ satisfy the following recurrence relation for each $k=0, \ldots, m$ and $l=0, \ldots, m+1$ :

$$
\begin{aligned}
& x q^{M_{1}} q^{2(m-k)}[k+1] R_{k+1, l}^{m+1}+q^{M_{1}+M_{2}}[m-k+1] R_{k, l}^{m+1} \\
& \quad=q^{M_{2}} q^{2 l}[m-l+1] R_{k, l}^{m}+x q^{M_{1}+M_{2}}[l] R_{k, l-1}^{m} . \text { and } \\
& q^{M_{1}+M_{2}}[k+1] R_{k+1, l}^{m+1}+q^{M_{2}} q^{2 k}[m-k+1] R_{k, l}^{m+1} \\
& \quad=q^{M_{1}+M_{2}}[m-l+1] R_{k, l}^{m}+q^{M_{1}} q^{2(m-l+1)}[l] R_{k, l-1}^{m} .
\end{aligned}
$$

Proof. Applying $\Delta^{\prime}\left(X_{0}^{+}\right)$to (2.2.5) and using $\Delta\left(X_{0}^{+}\right) R_{V_{1} V_{2}}(x)=R_{V_{1} V_{2}}(x) \Delta^{\prime}\left(X_{0}^{+}\right)$, we obtain

$$
\begin{aligned}
& R_{V_{1} V_{2}}(x)\left(x q^{-M_{2}+2(m-k)}[k+1] v^{(k+1)} \otimes v^{(m-k)}+[m-k+1] v^{(k)} \otimes v^{(m-k+1)}\right) \\
& =\sum_{l=0}^{m}\left(q^{-M_{1}+2 l}[m-l+1] R_{k, l}^{m} v^{(l)} \otimes v^{(m-l+1)}+x[l+1] R_{k, l}^{m} v^{(l+1)} \otimes v^{(m-l)}\right) \\
& =\sum_{l=0}^{m+1}\left(q^{-M_{1}+2 l}[m-l+1] R_{k, l}^{m}+x[l] R_{k, l-1}^{m}\right) v^{(l)} \otimes v^{(m-l+1)} .
\end{aligned}
$$

On the other hand, by definition,

$$
\begin{aligned}
& R_{V_{1} V_{2}}(x) v^{(k)} \otimes v^{(m-k+1)}=\sum_{l=0}^{m+1} R_{k, l}^{m+1} v^{(l)} \otimes v^{(m-l+1)}, \\
& R_{V_{1} V_{2}}(x) v^{(k+1)} \otimes v^{(m-k)}=\sum_{l=0}^{m+1} R_{k+1, l}^{m+1} v^{(l)} \otimes v^{(m-l)} .
\end{aligned}
$$

Substitute (2.2.9) in (2.2.8) and compare the coefficients to $v^{(l)} \otimes v^{(m-l+1)}$. Then we obtain (2.2.6). Similarly applying $\Delta^{\prime}\left(X_{1}^{-}\right)$to (2.2.5), we arrive at (2.2.7). Q.E.D.

Therefore $R_{k l}^{m}$ are uniquely determined by the scalar $R_{00}^{0}$ for general $q, \lambda_{1}$ and $\lambda_{2}$. We fix the normalization by $R_{00}^{0}=1$. Then, as a result, all $R_{k l}^{m}(x)$ are expressed by some rational functions, which can be continued with respect to the parameters. Thus, for arbitrary $q, \lambda_{i}$ and $\lambda_{j}$, we have uniquely specified the operator $R_{V_{i} V_{j}}(x)$ satisfying (2.1.6) and (2.1.7), whose matrix elements are rational functions in $x$.

2.3. Let $\lambda_{0}, \ldots, \lambda_{n}, \lambda_{\infty}$ be a set of weights such that

$$
\lambda_{0}+\cdots+\lambda_{n}-\lambda_{\infty}=m \alpha,
$$

where $m$ is a non-negative integer. Let $V_{i}$ be the irreducible representation of $U_{q}$ with the highest weight $\lambda_{i}$ and the highest weight vector $v_{i}$. Let $\mathscr{H}_{\lambda \infty}\left(V_{0} \otimes \cdots \otimes V_{n}\right)$ denote the set of the highest weight vectors with the weight $\lambda_{\infty}:$

$$
\begin{aligned}
& \mathscr{H}_{\lambda \infty}\left(V_{0} \otimes \cdots \otimes V_{n}\right) \\
& \quad=\left\{v \in V_{0} \otimes \cdots \otimes V_{n} ; \Delta^{(n)}\left(X^{+}\right) v=0, \Delta^{(n)}(K) v=q^{\left(\lambda_{\infty}, \alpha\right)} v\right\} .
\end{aligned}
$$

Let us normalize the R-matrices by

$$
R_{V_{i} V_{j}}(x) v_{i} \otimes v_{j}=v_{i} \otimes v_{j},
$$


as in the last subsection. If $R_{V_{i} V_{j}}(x)=\sum_{d} R_{i}^{(d)}(x) \otimes R_{j}^{(d)}(x)$, then we let it act on $V_{0} \otimes \cdots \otimes V_{n}$ by $1 \otimes \cdots \otimes R_{i}^{(d)}(x) \otimes \cdots \otimes R_{j}^{(d)}(x) \otimes \cdots \otimes 1$, which is nontrivial only on $i^{\text {th }}$ and $j^{\text {th }}$ components of the tensor product. Then we have

$$
R_{V_{i} V_{j}}(x) R_{V_{j} V_{i}}\left(x^{-1}\right)=\mathrm{id} .
$$

We next consider, for a weight $\lambda$, the operator defined by:

$$
q^{\pi_{i}(\lambda)} v_{i}^{(k)}=q^{\left(\lambda, \lambda_{i}-k \alpha\right)} v_{i}^{(k)} .
$$

Let $q^{\pi_{i}(\lambda)}$ act on the $i^{\text {th }}$ component of $V_{0} \otimes \cdots \otimes V_{n}$.

Let $p$ be a complex parameter and suppose $p^{-v}=q$. Let $T_{k}$ denote the $p$-shift operator:

$$
T_{k} F\left(x_{1}, \ldots, x_{n}\right)=F\left(x_{1}, \ldots, p x_{k}, \ldots, x_{n}\right) .
$$

The quantum Knizhnik-Zamolodchikov equation ( $\mathrm{qKZ}$ ) is, in some convention, the following system of equations for a function $\mathscr{F}=\mathscr{F}\left(x_{1}, \ldots, x_{n}\right)$ valued in $\mathscr{H}_{\lambda \infty}\left(V_{0} \otimes \cdots \otimes V_{n}\right)$ :

$$
\begin{aligned}
T_{i} \mathscr{F}= & R_{V_{i} V_{i-1}}\left(\frac{p x_{i}}{x_{i-1}}\right) \ldots R_{V_{i} V_{0}}\left(\frac{p x_{i}}{x_{0}}\right) \\
& q^{\pi_{i}\left(\lambda_{\infty}+\alpha\right)-\left(\lambda_{\infty}, \lambda_{2}\right)} R_{V_{n} V_{i}}\left(\frac{x_{n}}{x_{i}}\right)^{-1} \ldots R_{V_{i+1} V_{i}}\left(\frac{x_{i+1}}{x_{i}}\right)^{-1} \mathscr{F}, \\
& i=1, \ldots, n .
\end{aligned}
$$

Let us express $\mathscr{F}$ as

$$
\mathscr{F}=\sum_{k=0}^{\infty} v_{0}^{(k)} \otimes \mathscr{F}_{k},
$$

where $\mathscr{F}_{k}$ is a function valued in the weight space of $V_{1} \otimes \cdots \otimes V_{n}$ with the weight $\lambda_{\infty}-\lambda_{0}-k \alpha$. The $\mathrm{qKZ}$ for $\mathscr{F}_{0}$ is the following:

$$
\begin{aligned}
T_{i} \mathscr{F}_{0}= & R_{V_{i} V_{i-1}}\left(\frac{p x_{i}}{x_{i-1}}\right) \ldots R_{V_{l} V_{1}}\left(\frac{p x_{i}}{x_{1}}\right) \\
& \times q^{\pi_{i}\left(\lambda_{0}+\lambda_{\infty}+\alpha\right)-\left(\lambda_{0}+\lambda_{\infty}, \lambda_{i}\right)} R_{V_{n} V_{i}}\left(\frac{x_{n}}{x_{i}}\right)^{-1} \ldots R_{V_{i+1} V_{i}}\left(\frac{x_{i+1}}{x_{i}}\right)^{-1} \mathscr{F}_{0}, \\
& i=1, \ldots, n .
\end{aligned}
$$

If $\mathscr{F}$ is a solution to (2.3.7) then $\mathscr{F}_{0}$ is a solution to (2.3.9). If the parameters are general then $\mathscr{F}$ is recovered from $\mathscr{F}_{0}$ since $\Delta^{(n)}\left(X^{+}\right) \mathscr{F}=0$, and two equations are equivalent.

Remark. If $v=\frac{1}{2(l+2)}$ for some positive integer $l$ and $V_{i}$ are finite dimensional representations, then the $\mathrm{qKZ}(2.3 .9)$ is satisfied, up to nomalization by a certain scalar factor, by the $n$-point function of $q$-vertex operators. Roughly speaking, it is given by

$$
\mathscr{F}_{0}=\left\langle v_{0}, \Psi_{1} \cdots \Psi_{n} v_{\infty}\right\rangle \text {, }
$$

where $\Psi_{i}$ is an operator of the following kind:

$$
\Psi_{i}: V\left(\mu_{i-1}\right) \rightarrow V\left(\mu_{i}\right) \hat{\otimes} V_{i}\left(x_{i}\right) .
$$


Here $V\left(\mu_{j}\right)$ denotes an integrable highest weight representation of $\hat{U}_{q}$ of fixed level $l$ with the classical highest weight $\mu_{j}$. The weight $\mu_{j}$ should be appropriately chosen, and $v_{0}$ and $v_{\infty}$ are the highest weight vectors of $V\left(\lambda_{0}\right)$ and $V\left(\lambda_{\infty}\right)$ respectively. See [FR] for detail.

\section{Action of Symmetric Group}

3.1. Let us consider the $m^{\text {th }}$ symmetric group $S_{m}$ which consists of all permutations of letters $\{1, \ldots, m\}$. It is generated by the simple transportations $\sigma_{i}$, $i=1, \ldots, m-1$ with the following fundamental relations:

$$
\sigma_{i}^{2}=\mathrm{id}, \quad \sigma_{i} \sigma_{i+1} \sigma_{i}=\sigma_{i+1} \sigma_{i} \sigma_{i+1} \quad \text { and } \sigma_{i} \sigma_{j}=\sigma_{j} \sigma_{i} \text { for }|i-j|>1 \text {. }
$$

The following lemma is well-known:

Lemma 3.1.1. Let $S$ be a subgroup of $S_{m}$ generated by some of $\sigma_{i}, i=1 \ldots, m-1$. Then there exists a subset $X$ (resp. $Y$ ) of $S_{m}$ such that any element of $S_{m}$ is uniquely expressed as $s x$ by $x \in X$ and $s \in S$ (resp. ys by $y \in Y$ and $s \in S$ ).

For example, let $S$ be $S_{m-1}$ as a permutation group acting on $\{1, \ldots, m-1\}$. Then we may take

$$
X=\left\{\sigma_{m-1} \cdots \sigma_{j} ; 1 \leqq j \leqq m\right\}
$$

3.2. Now fix a general constant $q \in \mathbf{C}$ and set

$$
D_{i j}=\frac{t_{i}-q^{2} t_{j}}{q^{2} t_{i}-t_{j}} .
$$

Let $\mathbf{C}\left(t_{1}, \ldots, t_{m}\right)$ be the set of all rational functions with indeterminates $t_{1}, \ldots, t_{m}$. For an $f\left(t_{1}, \ldots, t_{m}\right) \in \mathbf{C}\left(t_{1}, \ldots, t_{m}\right)$, we define

$$
\left(\sigma_{i} f\right)\left(t_{1}, \ldots, t_{m}\right)=f\left(t_{1}, \ldots, t_{i+1}, t_{i}, \ldots, t_{m}\right) D_{i i+1} .
$$

For instance, let $i$ be an integer such that $1 \leqq i \leqq m$. Then we have

$$
\begin{gathered}
\left(\sigma_{m-1} \ldots \sigma_{i} f\right)\left(t_{1}, \ldots, t_{m}\right)=f\left(t_{1}, \ldots, t_{i-1}, t_{m}, t_{i}, \ldots, t_{m-1}\right) \prod_{i \leqq j \leqq m+1} D_{j m}, \\
\left(\left(\sigma_{i} \cdots \sigma_{1}\right)\left(\sigma_{i+1} \cdots \sigma_{2}\right) \cdots\left(\sigma_{m-1} \cdots \sigma_{m-i}\right) f\right)\left(t_{1}, \ldots, t_{m}\right) \\
=f\left(t_{i+1}, \ldots, t_{m}, t_{1}, \ldots, t_{i}\right) \prod_{\substack{1 \leqq j \leqq i \\
i+1 \leqq k \leqq m}} D_{j k} .
\end{gathered}
$$

Lemma 3.2.1. The action of $\sigma_{i}$ defined above extends to a representation of the symmetric group $S_{m}$ on the space $\mathbf{C}\left(t_{1}, \ldots, t_{m}\right)$.

Proof. It suffices to show the fundamental relation (3.1.1), which is easily verified.

Q.E.D.

For any subgroup $S$ of $S_{m}$ and a rational function $f\left(t_{1}, \ldots, t_{m}\right)$, we denote

$$
\sum_{S} f\left(t_{1}, \ldots, t_{m}\right)=\sum_{w \in S}(w f)\left(t_{1}, \ldots, t_{m}\right) .
$$

The following lemma will be used in Sect. 5 . 
Lemma 3.2.2. Let $f\left(t_{1}, \ldots, t_{m}\right)$ be a rational function such that $w f=f$ holds for any $w \in S_{m}$. Suppose that $g\left(t_{1}, \ldots, t_{m}\right)=\prod_{1 \leqq i<j \leqq m}\left(q^{2} t_{i}-t_{j}\right) f\left(t_{1}, \ldots, t_{m}\right)$ is a polynomial. Then it is divisible by $\prod_{1 \leqq i<j \leqq m}^{m}\left(\bar{t}_{i}-\bar{t}_{j}\right)$.

Proof. Let $k$ and $l$ be integers such that $1 \leqq k<l \leqq m$. Let $w \in S_{m}$ be the transposition of letters $k$ and $l$. It is written as $w=\sigma_{k} \cdots \sigma_{l-2} \sigma_{l-1} \sigma_{l-2} \cdots \sigma_{k}$. Then we have

$$
\begin{aligned}
g\left(t_{w(1)}, \ldots, t_{w(m)}\right) & =-D_{k l} \prod_{k<i<l} D_{k i} D_{i l} \prod_{1 \leqq i<j \leqq m}\left(q^{2} t_{i}-t_{j}\right) f\left(t_{w(1)}, \ldots, t_{(m)}\right) \\
& =-\prod_{1 \leqq i<j \leqq m}\left(q^{2} t_{i}-t_{j}\right)(w f)\left(t_{1}, \ldots, t_{m}\right) \\
& =-\prod_{1 \leqq i<j \leqq m}\left(q^{2} t_{i}-t_{j}\right) f\left(t_{1}, \ldots, t_{m}\right) \\
& =-g\left(t_{1}, \ldots, t_{m}\right) .
\end{aligned}
$$

Therefore the polynomial $g$ is divisible by $\left(t_{k}-t_{l}\right)$ for each pair $(k, l)$. Q.E.D.

For example, we have

$$
\begin{aligned}
\prod_{1 \leqq i<j \leqq m}\left(q^{2} t_{i}-t_{j}\right) \sum_{S_{m}} 1 & =\prod_{1 \leqq i<j \leqq m}\left(t_{i}-t_{j}\right) \sum_{w \in S_{m}} q^{2 l(w)} \\
& =\prod_{1 \leqq i<j \leqq m}\left(t_{i}-t_{j}\right) \prod_{d=1}^{m}\left(1+q^{2}+\cdots+q^{2 d-2}\right),
\end{aligned}
$$

where $l(w)$ denote the length of $w \in S_{m}$.

\section{Jackson Integrals of A-type Root System}

4.1. Let $p$ be a fixed complex number such as $0<|p|<1$. Let us denote

$$
(a ; p)_{\infty}=\prod_{n=0}^{\infty}\left(1-a p^{n}\right) \text {. }
$$

Let $u$ be a single variable. The $q$-difference operator $\frac{d}{d_{p} u}$ is defined by

$$
\frac{d}{d_{p} u} \phi(u)=\frac{\phi(p u)-\phi(u)}{u(p-1)}
$$

For a value $s \in \mathbf{C}-\{0\}$ and for a function $\phi(u)$, we define

$$
\int_{0}^{s \infty} \phi(u) \frac{d_{p} u}{u}=(1-p) \sum_{n=-\infty}^{\infty} \phi\left(s p^{n}\right)
$$

whenever it is convergent. This is called the Jackson integral along a $q$-interval $[0, s \infty]$, which is a $q$-difference analogue of the ordinary integration.

Let $t=\left(t_{1}, \ldots, t_{m}\right)$ be a multi-variable. The multiple Jackson integral, written as

$$
\int \phi(t) \frac{d_{p} t}{t}=\int \phi\left(t_{1}, \ldots, t_{m}\right) \frac{d_{p} t_{1}}{t_{1}} \ldots \frac{d_{p} t_{m}}{t_{m}},
$$


is similarly defined where a certain notion of $q$-cycles takes place instead of $q$-intervals (cf. [A2]). However we only need the following property:

$$
\int t_{k} \frac{d}{d_{p} t_{k}} \phi(t) \frac{d_{p} t}{t}=0 \quad \text { for any } 1 \leqq k \leqq m .
$$

This is a $q$-analogue of the Green-Stokes theorem. We do not discuss a choice of a $q$-cycle in this paper.

Let $\sim$ be the equivalence relation of functions defined as follows: $\phi_{1}\left(t_{1}, \ldots, t_{m}\right) \sim \phi_{2}\left(t_{1}, \ldots, t_{m}\right)$ if and only if there exists a function $\psi\left(t_{1}, \ldots, t_{m}\right)$ such that

$$
\phi_{1}\left(t_{1}, \ldots, t_{m}\right)-\phi_{2}\left(t_{1}, \ldots, t_{m}\right)=t_{i} \frac{d}{d_{p} t_{i}} \psi\left(t_{1}, \ldots, t_{m}\right)
$$

for some $i=1, \ldots, m$. Then if $\phi_{1}(t) \sim \phi_{2}(t)$ we have, by (4.1.5),

$$
\int \phi_{1}(t) \frac{d_{p} t}{t}=\int \phi_{2}(t) \frac{d_{p} t}{t} .
$$

4.2. Now, under the notations of 2.3 , we put

$$
M_{i}=\left(\lambda_{i}, \alpha\right) \text { and } M=\left(\lambda_{0}+\lambda_{\infty}+\alpha, \alpha\right) .
$$

Then, by the assumption (2.3.1), we have

$$
M=2 M_{0}+M_{1}+\cdots+M_{n}-2(m-1) .
$$

We define

$$
\begin{aligned}
\Phi_{p}(x, t)= & \prod_{\substack{1 \leqq i \leqq n \\
1 \leqq j \leqq m}}\left(\frac{x_{i}}{t_{j}}\right)^{-M_{i} v} \frac{\left(p^{M_{i} v} t_{j} / x_{i} ; p\right)_{\infty}}{\left(p^{-M_{i} v} t_{j} / x_{i} ; p\right)_{\infty}} \\
& \times \prod_{1 \leqq i<j \leqq m}\left(\frac{t_{j}}{t_{i}}\right)^{2 v} \frac{\left(p^{-2 v} t_{i} / t_{j} ; p\right)_{\infty}}{\left(p^{2 v} t_{i} / t_{j} ; p\right)_{\infty}} \prod_{1 \leqq i \leqq n} x_{i}^{-M_{i v} v} \prod_{1 \leqq j \leqq m} t_{j}^{M v} .
\end{aligned}
$$

Since $p^{-v}=q$, the definition (4.2.3) is rewritten as

$$
\begin{aligned}
\Phi_{p}(x, t)= & \prod_{\substack{1 \leqq i \leqq n \\
1 \leqq j \leqq m}}\left(\frac{x_{i}}{t_{j}}\right)^{-M_{i} v} \frac{\left(q^{-M_{i}} t_{j} / x_{i} ; p\right)_{\infty}}{\left(q^{M_{i}} t_{j} / x_{i} ; p\right)_{\infty}} \\
& \times \prod_{1 \leqq i<j \leqq m}\left(\frac{t_{j}}{t_{i}}\right)^{2 v} \frac{\left(q^{2} t_{i} / t_{j} ; p\right)_{\infty}}{\left(q^{-2} t_{i} / t_{j} ; p\right)_{\infty}} \prod_{1 \leqq i \leqq n} x_{i}^{-M_{i} v} \prod_{1 \leqq j \leqq m} t_{j}^{M v} .
\end{aligned}
$$

Now recall the action of the symmetric group (3.2.2). We have the following important lemma, which asserts that our action of $S_{m}$ is compatible with the Green-Stokes theorem twisted by $\Phi_{p}(x, t)$.

Lemma 4.2.1. If rational functions $\varphi_{1}(t)$ and $\varphi_{2}(t)$ satisfy $\Phi_{p}(x, t) \varphi_{1}(t) \sim$ $\Phi_{p}(x, t) \varphi_{2}(t)$, then $\Phi_{p}(x, t) w \varphi_{1}(t) \sim \Phi_{p}(x, t) w \varphi_{2}(t)$ holds for any $w \in S_{m}$.

Proof. It suffices to show the following assertion. Let $\varphi(t)=\varphi\left(t_{1}, \ldots, t_{m}\right)$ be a rational function. Then, for any $w \in S_{m}$ and any $1 \leqq i \leqq m$, we have:

$$
w\left(t_{i} \frac{d}{d_{p} t_{i}}\left\{\Phi_{p}(x, t) \varphi(t)\right\} / \Phi_{p}(x, t)\right)=t_{w^{-1}(i)} \frac{d}{d_{p} t_{w^{-1}(i)}}\left\{\Phi_{p}(x, t)(w \varphi)(x, t)\right\} / \Phi_{p}(x, t) .
$$


It is easily checked by direct calculations for each $w=\sigma_{1}, \ldots, \sigma_{n-1}$ using the following:

$$
\begin{gathered}
t_{i} \frac{d}{d_{p} t_{i}}\left\{\left(\frac{t_{j}}{t_{i}}\right)^{2 v} \frac{\left(q^{2} t_{i} / t_{j} ; p\right)_{\infty}}{\left(q^{-2} t_{i} / t_{j} ; p\right)_{\infty}}\right\}=\frac{D_{i j}-1}{p-1}\left(\frac{t_{j}}{t_{i}}\right)^{2 v} \frac{\left(q^{2} t_{i} / t_{j} ; p\right)_{\infty}}{\left(q^{-2} t_{i} / t_{j} ; p\right)_{\infty}}, \\
t_{j} \frac{d}{d_{p} t_{j}}\left\{\left(\frac{t_{j}}{t_{i}}\right)^{2 v} \frac{\left(q^{2} t_{i} / t_{j} ; p\right)_{\infty}}{\left(q^{-2} t_{i} / t_{j} ; p\right)_{\infty}} D_{i j}\right\}=\frac{1-D_{i j}\left(\frac{t_{j}}{t_{i}}\right)^{2 v} \frac{\left(q^{2} t_{i} / t_{j} ; p\right)_{\infty}}{\left(q^{-2} t_{i} / t_{j} ; p\right)_{\infty}} .}{} .
\end{gathered}
$$

4.3. We formally put $x_{0}=\infty$. For any $0 \leqq i \leqq n$ and $1 \leqq j \leqq m$, we set

$$
A_{i j}=\frac{q^{-M_{i}} x_{i}-t_{j}}{x_{i}-q^{-M_{i}} t_{j}}, \quad B_{i j}=\frac{x_{i}}{x_{i}-q^{-M_{i}} t_{j}} .
$$

Let $\vec{k}=\left(k_{0}, \ldots, k_{n}\right) \in\left(\mathbf{Z}_{\geqq 0}\right)^{n+1}$ be a multi-index such that $k_{0}+\cdots+k_{n}=m$ and define

$$
\vec{k} \cdot \vec{k}=\sum_{0 \leqq i<j \leqq n} k_{i} k_{j} .
$$

Putting $a_{i}=k_{0}+\cdots+k_{i}$, we set

$$
\begin{aligned}
& \varphi_{\vec{k}}(x, t)=q^{\vec{k} \cdot \vec{k}} \prod_{i=0}^{n}\left(\prod_{a_{i-1}+1 \leqq j \leqq a_{i}} A_{0 j} \cdots A_{i-1 j} B_{i j}\right), \\
& \tilde{\varphi}_{\vec{k}}(x, t)=\sum_{S_{m}} \varphi_{\vec{k}}(x, t) .
\end{aligned}
$$

We are interested in linear relations and $q$-difference equations among the Jackson integrals

$$
F_{\vec{k}}(x)=\int \Phi_{p}(x, t) \tilde{\varphi}_{\vec{k}}(x, t) \frac{d_{p} t}{t} .
$$

Remark. The definition of $A_{i j}$ originates in the following observation:

$$
t_{j} \frac{d}{d_{p} t_{j}}\left\{\left(\frac{x_{i}}{t_{j}}\right)^{-M_{i} v} \frac{\left(q^{-M_{i}} t_{j} / x_{i} ; p\right)_{\infty}}{\left(q^{M_{i}} t_{j} / x_{i} ; p\right)_{\infty}}\right\}=\frac{A_{i j}-1}{p-1}\left(\frac{x_{i}}{t_{j}}\right)^{-M_{i} v} \frac{\left(q^{-M_{i}} t_{j} / x_{i} ; p\right)_{\infty}}{\left(q^{M_{i}} t_{j} / x_{i} ; p\right)_{\infty}} .
$$

Note that $B_{i j}$ is related to $A_{i j}$ by the following equality:

$$
A_{i j}=\left(q^{-M_{i}}-q^{M_{i}}\right) B_{i j}+q^{M_{i}} .
$$

4.4. In this subsection, we will prove linear relations among $F_{\vec{k}}$.

Let $\vec{\varepsilon}_{i} \in\left(\mathbf{Z}_{\geqq 0}\right)^{n+1}$ be the multi-index with only non-zero entry at $i^{\text {th }}$ component.

Proposition 4.4.1. Let $\vec{k}^{\prime}=\left(k_{0}^{\prime}, \ldots, k_{n}^{\prime}\right)$ be a multi-index such that $k_{0}^{\prime}+\cdots+k_{n}^{\prime}$ $=m-1$. Then we have:

$$
\sum_{i=0}^{n}\left(q^{M_{i}-k_{i}^{\prime}}-q^{-M_{\imath}+k_{\imath}^{\prime}}\right) q^{-\left(M_{i+1}+\cdots+M_{n}\right)+2\left(k_{i+1}^{\prime}+\cdots+k_{n}^{\prime}\right)} F_{\vec{k}^{\prime}+\vec{\varepsilon}_{i}}=0 .
$$

Note. This relation is a $q$-deformation of Aomoto's relation (0.6) in [A1].

To prove this proposition, we prepare the following lemma:

Lemma 4.4.2. Let $S_{k+1}$ act on $\mathbf{C}\left(t_{1}, \ldots, t_{k+1}\right)$ as in 3.2. Then

$$
\begin{aligned}
\sum_{S_{k+1}}\left(q^{2 k} A_{i k+1}-q^{M_{i}}\right. & \left.\prod_{1 \leqq j \leqq k} D_{j k+1}\right) \prod_{1 \leqq j \leqq k} B_{i j} \\
& =q^{k}\left(q^{-M_{\imath}+k}-q^{M_{i}-k}\right) \sum_{S_{k+1}} \prod_{1 \leqq j \leqq k+1} B_{i j} .
\end{aligned}
$$


Proof. Because of (4.3.5), the left-hand side of (4.4.1) equals to

$$
q^{2 k}\left(q^{-M_{i}}-q^{M_{i}}\right) \sum_{S_{k+1}} \prod_{1 \leqq j \leqq k+1} B_{i j}+q^{M_{\imath}} \sum_{S_{k+1}}\left(q^{2 k}-\prod_{1 \leqq j \leqq k} D_{j k+1}\right) \prod_{1 \leqq j \leqq k} B_{i j}
$$

The second sum becomes

$$
\begin{aligned}
& q^{M_{i}} \sum_{S_{k+1}} B_{i k+1}^{-1}\left(q^{2 k}-\prod_{1 \leqq j \leqq k} D_{j k+1}\right) \prod_{1 \leqq j \leqq k+1} B_{i j} \\
& =\sum_{S_{k+1}}\left(-\frac{t_{k+1}}{x_{i}}+q^{M_{i}}\right)\left(q^{2 k}-\prod_{1 \leqq j \leqq k} D_{j k+1}\right) \prod_{1 \leqq j \leqq k+1} B_{i j} \\
& =\sum_{S_{k+1}}\left\{-\frac{t_{k+1}}{x_{i}}\left(q^{2 k}-\prod_{1 \leqq j \leqq k} D_{j k+1}\right)+q^{M_{i}}\left(q^{2 k}-\prod_{1 \leqq j \leqq k} D_{j k+1}\right)\right\} \prod_{1 \leqq j \leqq k+1} B_{i j} \\
& =-\frac{1}{x_{i}} \sum_{S_{k+1}}\left(q^{2 k} t_{k+1}-t_{1}\right) \prod_{1 \leqq j \leqq k+1} B_{i j}+q^{M_{i}} \sum_{S_{k+1}}\left(q^{2 k}-1\right) \prod_{1 \leqq j \leqq k+1} B_{i j} .
\end{aligned}
$$

Here we have used (3.2.3) in the last equality. Now we have

$$
q^{2 k} t_{k+1}-t_{1}=\sum_{j=1}^{k} q^{2 j-2}\left(q^{2} t_{j+1}-t_{j}\right)
$$

Since each summand is anti-symmetric by the action of $\sigma_{j}$, its summation with respect to $S_{k+1}$ is zero. Therefore (4.4.2) is written as

$$
\left\{q^{2 k}\left(q^{-M_{i}}-q^{M_{\imath}}\right)+q^{M_{\imath}}\left(q^{2 k}-1\right)\right\} \sum_{S_{k+1}} \prod_{1 \leqq j \leqq k+1} B_{i j},
$$

which is equal to the right-hand side of (4.4.1).

Q.E.D.

Proof of Proposition. We start with the identity

$$
\int t_{m} \frac{d}{d_{p} t_{m}}\left(\Phi_{p}(x, t) \tilde{\varphi}_{\vec{k}^{\prime}}(x, t) \prod_{1 \leqq j \leqq m-1} D_{j m}\right) \frac{d_{p} t}{t}=0,
$$

where $\tilde{\varphi}_{\vec{k}^{\prime}}(x, t)=\sum_{s_{m-1}} \varphi_{\vec{k}^{\prime}}\left(x, t_{1}, \ldots, t_{m-1}\right)$ is defined similarly as $\tilde{\varphi}_{\vec{k}}(x, t)$ provided the summation is over $S_{m-1}$ acting on $\mathbf{C}\left(t_{1}, \ldots, t_{m-1}\right)$. We set $a_{i}^{\prime}=k_{0}^{\prime}+\cdots+k_{i}^{\prime}$. By the definition of $\Phi_{p}(x, t)$, the integrand is calculated as

$$
\begin{aligned}
(p- & 1) t_{m} \frac{d}{d_{p} t_{m}}\left\{\Phi_{p}(x, t) \tilde{\varphi}_{\vec{k}^{\prime}}(x, t) \prod_{1 \leqq j \leqq m-1} D_{j m}\right\} \\
= & \Phi_{p}(x, t) \tilde{\varphi}_{\vec{k}^{\prime}}(x, t)\left(q^{2\left(k_{0}^{\prime}+\cdots+k_{n}^{\prime}\right)-\left(M_{0}+\cdots+M_{n}\right)} \prod_{0 \leqq i \leqq n} A_{i m}-\prod_{1 \leqq j \leqq m-1} D_{j m}\right) \\
= & \Phi_{p}(x, t) \tilde{\varphi}_{\vec{k}^{\prime}}(x, t) \sum_{i=0}^{n} q^{2\left(k_{0}^{\prime}+\cdots+k_{i-1}^{\prime}\right)-\left(M_{0}+\cdots+M_{i}\right)} \\
& \times\left(q^{2 k_{i}^{\prime}} A_{i m}-q^{M_{i}} \prod_{a_{i-1}^{\prime}+1 \leqq j \leqq a_{i}^{\prime}} D_{j m}\right)_{1 \leqq h \leqq i-1} \prod_{a_{i}^{\prime}+1 \leqq j \leqq m-1} A_{j m},
\end{aligned}
$$


which is $\sim 0$. Put $b_{i}=2\left(k_{0}^{\prime}+\cdots+k_{i-1}^{\prime}\right)-\left(M_{0}+\cdots+M_{i}\right)$ for simplicity. Similar calculation holds for

$$
\int t_{k} \frac{d}{d_{p} t_{k}}\left\{\Phi_{p}(x, t)\left(\sigma_{m-1} \ldots \sigma_{i} \tilde{\varphi}_{\vec{k}^{\prime}}\right)(x, t) \prod_{i \leqq j \leqq k-1} D_{j k}\right\} \frac{d_{p} t}{t}=0 .
$$

Summing them up, we can write the coefficient to $\Phi_{p}(x, t)$ as

$$
\begin{aligned}
\sum_{S_{m}} \sum_{i=0}^{n} q^{b_{i}} \varphi_{\vec{k}^{\prime}}(x, t)\left(q^{2 k_{i}^{\prime}} A_{i m}-q^{M_{i}} \prod_{a_{i-1}^{\prime}+1 \leqq j \leqq a_{t}^{\prime}} D_{j m}\right) \prod_{0 \leqq h \leqq i-1} A_{h m} \prod_{a_{i}^{\prime}+1 \leqq j \leqq m-1} D_{j m} \\
=\sum_{i=0}^{n} \sum_{S_{m}} q^{b_{i}}\left(\sigma_{1} \ldots \sigma_{a_{i}^{\prime}+1} \varphi_{\vec{k}^{\prime}}\right)(x, t)\left(q^{2 k_{i}^{\prime}} A_{i a_{i}^{\prime}+1}-q^{M_{\imath}} \prod_{a_{i-1}^{\prime}+1 \leqq j \leqq a_{i}^{\prime}} D_{j a_{i}^{\prime}+1}\right) \\
\quad \times \prod_{0 \leqq h \leqq i-1} A_{h a_{i}^{\prime}+1} .
\end{aligned}
$$

Using Lemma 3.1.1 and Lemma 4.4.2, we rewrite it as:

$$
\begin{aligned}
& \sum_{i=0}^{n} \sum_{S_{m}} q^{b_{i}}\left(\sigma_{1} \ldots \sigma_{a_{i}^{\prime}} \varphi_{\vec{k}^{\prime}}\right)(x, t) q^{k_{i}^{\prime}}\left(q^{-M_{i}+k_{i}^{\prime}}-q^{M_{i}-k_{i}^{\prime}}\right) B_{i a_{i}^{\prime}+1} \prod_{0 \leqq h \leqq i-1} A_{h a_{i}^{\prime}+1} \\
& =\sum_{i=0}^{n} q^{b_{i}+k_{i}^{\prime}}\left(q^{-M_{\imath}+k_{\imath}^{\prime}}-q^{M_{i}-k_{i}^{\prime}}\right) q^{\vec{k}^{\prime} \cdot \vec{k}^{\prime}} q^{-\left(\vec{k}^{\prime}+\vec{\varepsilon}_{i}\right) \cdot\left(\vec{k}^{\prime}+\vec{\varepsilon}_{\imath}\right)} \tilde{\varphi}_{\vec{k}^{\prime}}+\vec{\varepsilon}_{i}(x, t) \\
& =q^{-\left(k_{0}^{\prime}+\cdots+k_{n}^{\prime}\right)} \sum_{i=0}^{n} q^{2\left(k_{0}^{\prime}+\cdots+k_{i}^{\prime}\right)-\left(M_{0}+\cdots+M_{i}\right)}\left(q^{-M_{\imath}+k_{i}^{\prime}}-q^{\left.M_{i}-k_{i}^{\prime}\right)} \tilde{\varphi}_{\vec{k}^{\prime}+\vec{\varepsilon}_{i}}(x, t) .\right.
\end{aligned}
$$

Therefore we obtain

$$
\sum_{i=0}^{n}\left(q^{M_{i}-k_{i}^{\prime}}-q^{-M_{i}+k_{\imath}^{\prime}}\right) q^{\left(M_{i+1}+\cdots+M_{n}\right)-2\left(k_{i+1}^{\prime}+\cdots+k_{n}^{\prime}\right)} \Phi_{p}(x, t) \tilde{\varphi}_{\vec{k}^{\prime}+\vec{\varepsilon}_{t}}(x, t) \sim 0 .
$$

Q.E.D.

\section{Solutions of Quantum Knizhnik-Zamolodchikov Equation}

5.1. Let us interpret Proposition 4.4.1 in the language of $U_{q}$. We keep the notations in Sect. 2.

For a multi-index $\vec{k}=\left(k_{0}, \ldots, k_{n}\right)$, we set

$$
v_{\vec{k}}=v_{0}^{\left(k_{0}\right)} \otimes \cdots \otimes v_{n}^{\left(k_{n}\right)},
$$

where $v_{i}^{(k)}$ is defined by (2.2.2). We define a $V_{0} \otimes \cdots \otimes V_{n}$-valued function $\mathscr{F}$ by

$$
\mathscr{F}=\sum_{|\vec{k}|=m} F_{\vec{k}}(x) v_{\vec{k}},
$$

where $F_{\vec{k}}(x)$ is defined by (4.3.4) and the summation is over all multi-indices $\vec{k}$ such that $k_{0}+\cdots+k_{n}=m$. The weight of $\mathscr{F}$ is $\lambda_{\infty}$ because of (2.3.1).

Then, as a corollary to Proposition 4.4.1, we obtain

Proposition 5.1.1. The vector $\mathscr{F}$ satisfies $\Delta^{(n)}\left(X^{+}\right) \mathscr{F}=0$. Namely, $\mathscr{F}$ is a function valued in $\mathscr{H}_{\lambda_{\infty}}\left(V_{0} \otimes \cdots \otimes V_{n}\right)$. 
Conjecture 5.1.2. The function $\mathscr{F}$ is a solution of the $\mathrm{qKZ}$ :

$$
\begin{aligned}
& T_{i} \mathscr{F}=R_{V_{i} V_{\imath-1}}\left(\frac{p x_{i}}{x_{i-1}}\right) \cdots R_{V_{i} V_{0}}\left(\frac{p x_{i}}{x_{0}}\right) \\
& \times q^{\pi_{i}\left(\lambda_{\infty}+\alpha\right)-\left(\lambda_{\infty}, \lambda_{i}\right)} R_{V_{n} V_{i}}\left(\frac{x_{n}}{x_{i}}\right)^{-1} \cdots R_{V_{i+1} V_{i}}\left(\frac{x_{i+1}}{x_{i}}\right)^{-1} \mathscr{F} .
\end{aligned}
$$

If $m=1$ and $n$ is arbitrary, the conjecture is true [Ma2]. It is also true when $n=2$ and $m$ is arbitrary as we shall show in the next subsection.

5.2. We consider the case $n=2$. Let us denote

$$
\begin{aligned}
\varphi_{k}(x, t) & =\varphi_{(0, k, m-k)}(x, t) \\
& =q^{k(m-k)} \prod_{1 \leqq j \leqq k} B_{1 j} \prod_{k+1 \leqq j \leqq m} A_{1 j} B_{2 j}, \\
& F_{k}(x)=F_{(0, k, m-k)}(x) .
\end{aligned}
$$

Then $\mathscr{F}_{0}$ is given by

$$
\mathscr{F}_{0}=\sum_{k=0}^{m} F_{k}(x) v_{1}^{(k)} \otimes v_{2}^{(m-k)} .
$$

Recall that $R_{V_{2} V_{1}}\left(p x_{2} / x_{1}\right) R_{V_{1} V_{2}}\left(x_{1} / p x_{2}\right)=\mathrm{id}$.

Theorem 5.2.1. The function $\mathscr{F}_{0}$ satisfies the $\mathrm{qKZ}$ :

$$
\begin{aligned}
& T_{1} \mathscr{F}_{0}=q^{\pi_{1}\left(\lambda_{0}+\lambda_{\infty}+\alpha\right)-\left(\lambda_{0}+\lambda_{\infty}, \lambda_{1}\right)} R_{V_{1} V_{2}}\left(\frac{x_{1}}{x_{2}}\right) \mathscr{F}_{0}, \\
& T_{2} \mathscr{F}_{0}=R_{V_{2} V_{1}}\left(\frac{p x_{2}}{x_{1}}\right) q^{\pi_{2}\left(\lambda_{0}+\lambda_{\infty}+\alpha\right)-\left(\lambda_{0}+\lambda_{\infty}, \lambda_{2}\right) \mathscr{F}_{0} .}
\end{aligned}
$$

Proof. Since we have

$$
\begin{aligned}
T_{2} T_{1} \Phi_{p} & (x, t) \tilde{\varphi}_{l}(x, t) \\
& \sim T_{2} T_{1} \Phi\left(x, p t_{1}, \ldots, p t_{m}\right) \tilde{\varphi}_{k}\left(x, p t_{1}, \ldots, p t_{m}\right) \\
& =q^{M_{1}+M_{2}-m M} \Phi_{p}(x, t) \tilde{\varphi}_{k}(x, t) \\
& =q^{\left(\pi_{1}+\pi_{2}\right)\left(\lambda_{0}+\lambda_{\infty}+\alpha\right)-\left(\lambda_{0}+\lambda_{\infty}, \lambda_{1}+\lambda_{2}\right)} \Phi_{p}(x, t) \tilde{\varphi}_{k}(x, t),
\end{aligned}
$$

it holds that

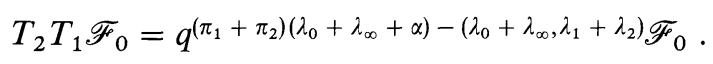

Therefore (5.2.5) follows from (5.2.4). To show (5.2.4) it suffices to verify that the functions $R_{k, l}^{m}\left(x_{1} / x_{2}\right)$, which are the matrix elements of $R_{V_{1} V_{2}}\left(x_{1} / x_{2}\right)$, satisfy the following:

$$
T_{1} \Phi_{p}(x, t) \tilde{\varphi}_{l}(x, t) \sim \sum_{k=0}^{m} q^{M_{1}-l M} R_{k, l}^{m}\left(x_{1} / x_{2}\right) \Phi_{p}(x, t) \tilde{\varphi}_{k}(x, t) .
$$


Now we have

$$
\begin{aligned}
T_{1} \Phi_{p}(x, t) \varphi_{l}(x, t) & \\
& \sim T_{1} \Phi\left(x, p t_{1}, \ldots, p t_{l}, t_{l+1}, \ldots, t_{m}\right) \varphi_{l}\left(x, p t_{1}, \ldots, p t_{l}, t_{l+1}, \ldots, t_{m}\right) \\
& =q^{M_{1}-l M} q^{l(m-l)} \Phi_{p}(x, t) \prod_{1 \leqq j \leqq l} B_{1 j} A_{2 j} \prod_{l+1 \leqq j \leqq m} B_{2 j} \prod_{\substack{1 \leqq i \leqq l \\
l+1 \leqq j \leqq m}} D_{i j} .
\end{aligned}
$$

Therefore by Lemma 4.2.1 it is sufficient to prove

$$
\begin{aligned}
q^{l(m-l)} & \sum_{S_{m}} \prod_{1 \leqq j \leqq l} B_{1 j} A_{2 j} \prod_{l+1 \leqq j \leqq m} B_{2 j} \prod_{\substack{1 \leqq i \leqq l \\
l+1 \leqq j \leqq m}} D_{i j} \\
& =\sum_{k=0}^{m} R_{k, l}^{m}\left(x_{1} / x_{2}\right) q^{k(m-k)} \sum_{S_{m}} \prod_{1 \leqq j \leqq k} B_{1 j} \prod_{k+1 \leqq j \leqq m} A_{i j} B_{2 j} .
\end{aligned}
$$

By (3.2.4), the left-hand side of (5.2.7) is written as

$$
q^{l(m-l)} \sum_{S_{m}} \prod_{m-l+1 \leqq j \leqq m} B_{1 j} A_{2 j} \prod_{1 \leqq j \leqq m-l} B_{2 j} .
$$

Then having multiplied by some of common denominators, the proof of (5.2.7) reduces to the following lemma:

Lemma 5.2.2. The functions $S_{k, l}^{m}=R_{k, l}^{m}\left(x_{1} / x_{2}\right)$ satisfy the identity

$$
\begin{aligned}
& q^{l(m-l)} \sum_{S_{m}} \prod_{m-l+1 \leqq j \leqq m} x_{1}\left(q^{-M_{2}} x_{2}-t_{j}\right) \prod_{1 \leqq j \leqq m-l} x_{2}\left(x_{1}-q^{-M_{1}} t_{j}\right) \\
& \quad=\sum_{k=0}^{m} S_{k, l}^{m} q^{k(m-k)} \sum_{S_{m}} \prod_{1 \leqq j \leqq k} x_{1}\left(x_{2}-q^{-M_{2}} t_{j}\right) \prod_{k+1 \leqq j \leqq m} x_{2}\left(q^{-M_{1}} x_{1}-t_{j}\right) .
\end{aligned}
$$

Proof. To simplify expressions below, we put

$$
\begin{aligned}
K_{k}^{m} & =\sum_{S_{m}} \prod_{1 \leqq j \leqq k} x_{1}\left(x_{2}-q^{-M_{2}} t_{j}\right) \prod_{k+1 \leqq j \leqq m} x_{2}\left(q^{-M_{1}} x_{1}-t_{j}\right), \\
L_{l}^{m} & =\sum_{S_{m}} \prod_{1 \leqq j \leqq m-l} x_{2}\left(x_{1}-q^{-M_{1}} t_{j}\right) \prod_{m-l+1 \leqq j \leqq m} x_{1}\left(q^{-M_{2}} x_{2}-t_{j}\right) .
\end{aligned}
$$

Then (5.2.8) becomes

$$
q^{l(m-l)} L_{l}^{m}=\sum_{k=0}^{m} S_{k, l}^{m} q^{k(m-k)} K_{k}^{m} .
$$

We shall prove by induction on $m$ that $S_{k, l}^{m}=R_{k, l}^{m}\left(x_{1} / x_{2}\right)$ satisfy (5.2.9). For $m=0$, it trivially holds by the normalization (2.3.4). We assume that (5.2.9) holds for $m-1$. First notice the following observation. Multiply both sides of (5.2.8) by

$$
\prod_{1 \leqq i<j \leqq m} \frac{q^{2} t_{i}-t_{j}}{t_{i}-t_{j}} .
$$


Then it follows from Lemma 3.2.2 that the results are polynomials whose degree is one with respect to each $t_{i}, i=1, \ldots, m$. Therefore it suffices to verify the equality at two different values of each $t_{i}$. We will consider the limits $t_{m} \rightarrow 0$ and $t_{m} \rightarrow \infty$. Now let $S_{m-1}$ acts on $\mathbf{C}\left(t_{1}, \ldots, t_{m-1}\right)$. For any function $f\left(t_{1}, \ldots, t_{m}\right)$, we have

$$
\begin{aligned}
\sum_{S_{m}} f\left(t_{1}, \ldots, t_{m}\right) & =\sum_{S_{m-1}} \sum_{i=1}^{m}\left(\sigma_{m-1} \cdots \sigma_{i} f\right)\left(t_{1}, \ldots, t_{m}\right) \\
& =\sum_{i=1}^{m} \sum_{S_{m-1}} f\left(t_{1}, \ldots, t_{i-1}, t_{m}, t_{i}, \ldots, t_{m-1}\right) \prod_{1 \leqq j \leqq m-1} D_{j m}
\end{aligned}
$$

by (3.1.2). Therefore, letting $t_{m} \rightarrow 0$ and $t_{m} \rightarrow \infty$, it becomes

$$
\begin{gathered}
\sum_{i=1}^{m} \sum_{S_{m-1}} f\left(t_{1}, \ldots, t_{i-1}, 0, t_{i}, \ldots, t_{m-1}\right) q^{-2(m-i)} \text { and } \\
\quad \sum_{i=1}^{m} \sum_{S_{m-1}} f\left(t_{1}, \ldots, t_{i-1}, \infty, t_{i}, \ldots, t_{m-1}\right) q^{2(m-i)}
\end{gathered}
$$

respectively. Applying this consideration to $K_{k}^{m}$, we obtain

$$
\begin{aligned}
\lim _{t_{m} \rightarrow 0} K_{k}^{m} & =x_{1} x_{2}\left(\sum_{i=1}^{k} q^{-2(m-i)}\right) K_{k-1}^{m-1}+q^{-M_{1}} x_{1} x_{2}\left(\sum_{i=k+1}^{m} q^{-2(m-i)}\right) K_{k}^{m-1} \\
& =x_{1} x_{2}\left(q^{-2(m-k)} q^{-(k-1)}[k] K_{k-1}^{m-1}+q^{-M_{1}} q^{-(m-k-1)}[m-k] K_{k}^{m-1}\right), \\
\lim _{t_{m} \rightarrow \infty} \frac{1}{t_{m}} K_{k}^{m} & =-q^{-M_{2}} x_{1}\left(\sum_{i=1}^{k} q^{2(m-i)}\right) K_{k-1}^{m-1}-x_{2}\left(\sum_{i=k+1}^{m} q^{2(m-i)}\right) K_{k}^{m-1} \\
& =-x_{1} q^{-M_{2}} q^{2(m-k)} q^{k-1}[k] K_{k-1}^{m-1}-x_{2} q^{m-k-1}[m-k] K_{k}^{m-1} .
\end{aligned}
$$

Similarly applying to $L_{l}^{m}$, we get

$$
\begin{aligned}
\lim _{t_{m} \rightarrow 0} L_{l}^{m} & =x_{1} x_{2}\left(q^{-2 l} q^{-(m-l-1)}[m-l] L_{l}^{m-1}+q^{-M_{2}} q^{-(l-1)}[l] L_{l-1}^{m-1}\right), \\
\lim _{t_{m} \rightarrow \infty} \frac{1}{t_{m}} L_{l}^{m} & =-x_{2} q^{-M_{1}} q^{2 l} q^{m-l-1}[m-l] L_{l}^{m-1}-x_{1} q^{l-1}[l] L_{l-1}^{m-1} .
\end{aligned}
$$

Thus we see that $S_{k, l}^{m}$ satisfy (5.2.9) if and only if they satisfy both of the following two:

$$
\begin{aligned}
& q^{l(m-l)}\left(q^{-M_{2}} q^{-2(m-l)} q^{-(l-1)}[l] L_{l-1}^{m-1}+q^{-(m-l-1)}[m-l] L_{l}^{m-1}\right) \\
& =\sum_{k=0}^{m-1} K_{k}^{m-1}\left\{q^{-M_{1}} q^{k(m-k)} q^{-(m-k-1)}[m-k] S_{k, l}^{m}\right. \\
& \left.\quad+q^{(k+1)(m-k-1)} q^{-2(m-k-1)} q^{-k}[k+1] S_{k+1, l}^{m}\right\}, \\
& q^{l(m-l)}\left(x_{1} q^{l-1}[l] L_{l-1}^{m-1}+x_{2} q^{-M_{1}} q^{2 l} q^{m-l-1}[m-l] L_{l}^{m-1}\right) \\
& =\sum_{k=0}^{m-1} K_{k}^{m-1}\left\{x_{2} q^{k(m-k)} q^{m-k-1}[m-k] S_{k, l}^{m}\right. \\
& \left.\quad+x_{1} q^{-M_{2}} q^{(k+1)(m-k-1)} q^{2(m-k-1)} q^{k}[k+1] S_{k+1, l}^{m}\right\} .
\end{aligned}
$$


Now by the induction hypothesis, we have

$$
\begin{aligned}
& L_{l}^{m-1}=q^{-l(m-l-1)} \sum_{k=0}^{m-1} q^{k(m-k-1)} R_{k, l}^{m-1} K_{k}^{m-1}, \\
& L_{l-1}^{m-1}=q^{-(l-1)(m-l)} \sum_{k=0}^{m-1} q^{k(m-k-1)} R_{k, l-1}^{m-1} K_{k}^{m-1} .
\end{aligned}
$$

Substitute them in (5.2.10) and (5.2.11) and equate the coefficients to $K_{k}^{m-1}$. Then we see that the following equalities for all $k=0, \ldots, m-1$ are sufficient to the conditions (5.2.10) and (5.2.11):

$$
\begin{gathered}
q^{M_{2}} q^{2 k}[m-k] S_{k, l}^{m}+q^{M_{1}+M_{2}}[k+1] S_{k+1, l}^{m} \\
=q^{M_{1}} q^{2(m-l)}[l] R_{k, l-1}^{m-1}+q^{M_{1}+M_{2}}[m-l] R_{k, l}^{m-1}, \\
x_{2} q^{M_{1}+M_{2}}[m-k] S_{k, l}^{m}+x_{1} q^{M_{1}} q^{2(m-k-1)}[k+1] S_{k+1, l}^{m} \\
\quad=x_{1} q^{M_{1}+M_{2}}[l] R_{k, l-1}^{m-1}+x_{2} q^{M_{2}} q^{2 l}[m-l] R_{k, l}^{m-1} .
\end{gathered}
$$

Comparing them with the intertwining property (2.2.6) and (2.2.7), we see that $S_{k, l}^{m}=R_{k, l}^{m}\left(x_{1} / x_{2}\right)$ satisfy (5.2.9). Thus the induction is completed. Q.E.D.

Note. For general values of $q, \lambda_{1}$ and $\lambda_{2}$, the equality (5.2.8) characterizes the intertwiner $R_{V_{1} V_{2}}(x)$ with the normalization (2.3.3).

5.3. Remark. Put $x_{0}=0$ and $\hat{p}=p^{-1}$. We define the function $\hat{\mathscr{F}}$ similarly as $\mathscr{F}$, by substituting

$$
\begin{aligned}
\hat{\Phi}_{\hat{p}}(x, t)= & \prod_{\substack{1 \leqq i \leqq n \\
1 \leqq j \leqq m}}\left(\frac{x_{i}}{t_{j}}\right)^{-M_{i} v} \frac{\left(\hat{p}^{M_{i} v} t_{j} / x_{i} ; \hat{p}\right)_{\infty}}{\left(\hat{p}^{-M_{i} v} t_{j} / x_{i} ; \hat{p}\right)_{\infty}} \\
& \times \prod_{1 \leqq i<j \leqq m}\left(\frac{t_{j}}{t_{i}}\right)^{2 v} \frac{\left(\hat{p}^{-2 v} t_{i} / t_{j} ; \hat{p}\right)_{\infty}}{\left(\hat{p}^{2 v} t_{i} / t_{j} ; \hat{p}\right)_{\infty}} \prod_{1 \leqq i \leqq n} x_{i}^{M_{i} v} \prod_{1 \leqq j \leqq m} t_{j}^{-M_{v}-1},
\end{aligned}
$$

and

$$
\hat{A}_{i j}=\frac{q^{M_{i}} x_{i}-t_{j}}{x_{i}-q^{M_{i}} t_{j}} \quad \hat{B}_{i j}=\frac{-q^{M_{i}} t_{j}}{x_{i}-q^{M_{i}} t_{j}} \quad \hat{D}_{i j}=\frac{q^{2} t_{i}-t_{j}}{t_{i}-q^{2} t_{j}},
$$

instead of $\Phi_{p}(x, t), A_{i j}, B_{i j}$ and $D_{i j}$ in the definition of $\mathscr{F}$. Then we have $\left.\Delta^{(n)} \Phi X^{+}\right) \hat{\mathscr{F}}=0$. We conjecture that $\hat{\mathscr{F}}$ is a solution of the following $q$-difference equation:

$$
\begin{aligned}
\hat{T_{i}} \hat{\mathscr{F}}= & R_{V_{i} V_{i-1}}\left(\frac{x_{i-1}}{\hat{p} x_{i}}\right) \cdots R_{V_{i} V_{0}}\left(\frac{x_{0}}{\hat{p} x_{i}}\right) \\
& \times q^{\pi_{i}\left(\lambda_{\infty}+\alpha\right)-\left(\lambda_{\infty}, \lambda_{i}\right)} R_{V_{n} V_{i}}\left(\frac{x_{i}}{x_{n}}\right)^{-1} \cdots R_{V_{i+1} V_{i}}\left(\frac{x_{i}}{x_{i+1}}\right)^{-1} \hat{\mathscr{F}},
\end{aligned}
$$

where $\hat{T}_{i}$ denote the shift of $x_{i}$ by $\hat{p}$. This conjecture is true for $m=1$ or $n=2$. The proofs are similar to the case of $\mathscr{F}$. Therefore, by transforming the variables as $x_{i} \mapsto \frac{1}{x_{i}}$, we obtain another expression of solutions to $\mathrm{qKZ}$. 
Acknowledgements. The author is grateful to M. Jimbo and K. Aomoto for useful discussions and comments.

\section{References}

[ABF] Andrews, G.E., Baxter, R.J., Forrester, P.J.: Eight-vertex SOS model and generalized Rogers-Ramanujan-type identities. J. Stat. Phys. 35, 193-266 (1984)

[A1] Aomoto, K.: Gauss-Manin connection of integrals of difference products. J. Math. Soc. Japan 39, 191-208 (1987)

[A2] Aomoto, K.: Finiteness of a cohomology associated with certain Jackson integrals. Tohoku Math. J. 43, 75-101 (1991)

[AKM] Aomoto, K., Kato, Y., Mimachi, K.: A solution of Yang-Baxter equation as connection coefficients of a holonomic q-difference system. Duke Math. J. 65, IMRN 7-15 (1992)

[ATY] Awata, H., Tsuchiya, A., Yamada, Y.: Integral formulas for the WZNW correlation functions. Nucl. Phys. B365, 680-696

[Ch] Cherednik, I.V.: Integral solutions to the trigonometric Knizhnik-Zamolodchikov equations and Kac-Moody algebras. Publ. RIMS 27, 727-744 (1991)

[CF] Christe, P., Flume, R.: The four point correlators of all primary operators of the $d=2$ conformally invariant $S U(2) \sigma$-model with Wess-Zumino term. Nucl. Phys B282, 466-494 (1987)

[DJMM] Date, E., Jimbo, M., Matsuo, A., Miwa, T.: Hypergeometric type integrals and the sl $(2$, C) Knizhnik-Zamolodchikov equations. Int. J. Mod. Phys. B4, 1049 (1990)

[D] Drinfeld, V.G.: Quantum groups. In Proc. ICM, Berkeley, Providence, RI: AMS 1986

[FR] Frenkel, I.B., Reshetikhin, N.Yu.: Quantum affine algebras and holonomic difference equations. Commun. Math. Phys. 146, 1-60 (1992)

[J1] Jimbo, M.: A q-difference analogue of $U(\mathrm{~g})$ and the Yang-Baxter equation, Lett. Math. Phys. 10, 63-69 (1985)

[J2] Jimbo, M.: Quantum groups and Yang-Baxter equation. Berlin, Heidelberg, New York, Tokyo: Springer 1990 (in Japanese)

[JMO] Jimbo, M., Miwa, T., Okado, M.: Solvable lattice models related to the vector representation of classical simple Lie algebra. Commun. Math. Phys. 116, 507-525 (1988)

[KZ] Knizhnik, V.G., Zamolodchikov, A.B.: Current algebra and Wess-Zumino models in two dimensions. Nucl. Phys. B247, 83-103 (1984)

[KR] Kulish, P.P., Reshetikhin, N.Yu.: Quantum linear problem for the sine-Gordon equation and higher representations. Zapiski Nauch. Semin. LOMI 101, 101-110 (1980); J. Soviet Math. 23, 2435-2441 (1983)

[K] Kuroki, G.: Fock space representations of affine Lie algebras and integral representations in the Wess-Zumino-Witten model. Commun. Math. Phys. 142, 511-542 (1991)

[Ma1] Matsuo, A.: An application of Aomoto-Gelfand hypergeometric functions to $S U(n)$ Knizhnik-Zamolodchikov equation. Commun. Math. Phys. 134, 65-77 (1990)

[Ma2] Matsuo, A.: Jackson integrals of Jordan-Pochhammer type and quantum KnizhnikZamolodchikov equations. Commun. Math. Phys. 151, 263-273 (1993)

[Mi1] Mimachi, K.: Connection problem in holonomic $q$-difference system associated with a Jackson integral of Jordan-Pochhammer type. Nagoya Math. J. 116, 149-161 (1989)

[Mi2] Mimachi, K.: Holonomic $q$-difference system of the first order associated with the Jackson integral of Selberg type. Preprint (1992)

[R] Reshetikhin, N.Yu.: Jackson type integrals, Bethe vectors, and solutions to a difference analog of the Knizhnik-Zamolodchikov system. Preprint (1992)

[SV1] Schechtman, V.V., Varchenko, A.N.: Integral representation of $N$-point conformal correlators in the WZW model. Preprint Max-Planck-Institut für Mathematik MPI/89-51

[SV2] Schechtman, V.V., Varchenko, A.N.: Arrangement of hyperplanes and Lie algebra homology. Invent. Math. 106, 139-194 (1991) 
[T] Tanisaki, T.: Killing forms, Harish-Chandra isomorphisms, and universal Rmatrices for quantum algebras. Preprint (1991)

[TK] Tsuchiya, A., Kanie, Y.: Vertex operators in conformal field theory on $\mathbf{P}^{1}$ and monodromy representations of braid groups. Adv. Stud. Pure. Math. 16, 297-372 (1988)

[ZF] Zamolodchikov, A.B., Fateev, V.A.: Operator algebra and correlation functions in the two dimensional $S U(2) \times S U(2)$ chiral Wesss-Zumino model. Sov. J. Nucl. Phys. 43, 657-644 (1986); Yad. Fiz. 43, 1031-1044 (1986)

Communicated by H. Araki 